\title{
GEOMETRY OF A SURFACE IN THE NEIGHBORHOOD OF A SPINE*
}

\author{
BY \\ V. G. GROVE
}

1. Introduction. In this paper we propose to continue the study begun by Bompiani, $\uparrow$ Lane, and others of curves and surfaces in the neighborhood of a singularity. The curves and surfaces previously studied possessed a tangent line or plane at the singularity. We shall discuss from a projective point of view the geometry of a surface in the neighborhood of a singularity we have called a spine by making use of certain osculants of a plane curve at a cusp.

We shall say that a point $O$ on a surface $S$ is a spine if it is a conical point such that the cone at the conical point degenerates into two planes. A spine may also be defined as a point $O$ through which there exists a line $t$ such that every nonspecialized plane section of $S$ through $t$ has an ordinary cusp at $O$ with $t$ as the cuspidal tangent.

Two cases arise according as the degenerate cone consists of distinct planes or coincident planes. In the first case, which we will call the nonparabolic case, the line $t$ is unique and is the intersection of the distinct planes. We shall call the line $t$ the spinal tangent. In the second case, which we shall call the parabolic case, the line $t$ is any line in the pair of coincident planes. We shall call $t$ a spinal tangent, and shall call such a spine $O$ a spine of the parabolic type.

In $\$ \$ 3$ and 4 we derive the equations of certain osculating curves of a curve at a cusp. In particular, we define order of contact of an algebraic curve with a given curve at its cusp.

In $\$ 2$ we derive a simple form of the equation of a surface $S$ with a spine at $O$ in the nonparabolic case, and then use the osculants for a curve at a cusp to study the surface

In the last section we briefly discuss the surface in the neighborhood of a spine of the parabolic type.

2. The canonical form of the equation of $S$. Let the surface $S$ have a spine of the nonparabolic type at $O$. Let the nonhomogeneous projective co-

* Presented to the Society, April 10, 1937; received by the editors January 24, 1938.

$\dagger \mathrm{E}$. Bompiani, Per lo studio proiettivo-differenziale delle singolaritd, Bolletino dell' Unione Matematica, vol. 5 (1926), p. 118; E. P. Lane, The neighborhood of a sextactic point on a plane curve, Duke Mathematical Journal, vol. 1 (1935), p. 287; T. L. Downs, Asymptotic and principal directions at a planar point of a surface, Duke Mathematical Journal, vol. 1 (1935), p. 316; V. G. Grove, Differential geometry of a surface at a planar point, these Transactions, vol. 40 (1936), p. 155. 
ordinates of a point $P$ be $(x, y, z)$. If the planes $y=0, z=0$ be chosen as the distinct planes of which the degenerate cone consists, and the point $(0,0,0)$ be chosen at the spine, we may write the equation of the surface in the form

(1) $y z=A_{3}+B_{2} x+C_{1} x^{2}+D_{0} x^{3}+A_{4}+B_{3} x+C_{2} x^{2}+D_{1} x^{3}+E_{0} x^{4}+\cdots$, wherein $A_{i}, B_{i}, C_{i}, \cdots$ are homogeneous functions of degree $i$ in $y$ and $z$ only. By transformations of the form

$$
\begin{aligned}
x & =x^{\prime}+\alpha y^{\prime}+\beta z^{\prime}, \quad y=y^{\prime}, \quad z=z^{\prime} ; \\
x^{\prime} & =\frac{\bar{x}}{1+\lambda \bar{x}+\mu \bar{y}+\nu \bar{z}}, \quad y^{\prime}=\frac{\bar{y}}{1+\lambda \bar{x}+\mu \bar{y}+\nu \bar{z}}, \quad z^{\prime}=\frac{\bar{z}}{1+\lambda \bar{x}+\mu \bar{y}+\nu \bar{z}} ; \\
\bar{x} & =q \xi, \quad \bar{y}=r \eta, \quad \bar{z}=s \zeta,
\end{aligned}
$$

we may reduce equation (1) to the following canonical form

(2) $\eta \zeta=A_{3}+B_{2} \xi+C_{1} \xi^{2}+D_{0} \xi^{3}+A_{4}+B_{3} \xi+C_{2} \xi^{2}+D_{1} \xi^{3}+E_{0} \xi^{4}+\cdots$, wherein

$$
\begin{aligned}
& A_{3}=A_{30} \eta^{3}+A_{21} \eta^{2} \zeta+A_{12} \eta \zeta^{2}+A_{03} \zeta^{3}, \\
& B_{2}=-A_{30} \eta^{2}+B_{11} \eta \zeta-A_{03} \zeta^{2}, \\
& C_{1}=0 \\
& D_{0}=1 \\
& A_{4}=A_{40} \eta^{4}+A_{31} \eta^{3} \zeta+A_{22} \eta^{2} \zeta^{2}+A_{13} \eta \zeta^{3}+A_{04} \zeta^{4}, \\
& B_{3}=B_{30} \eta^{3}+B_{21} \eta^{2} \zeta+B_{12} \eta \zeta^{2}+B_{03} \zeta^{3}, \\
& C_{2}=C_{20} \eta^{2}+C_{11} \eta \zeta+C_{02} \zeta^{2}, \\
& D_{1}=-A_{21} \eta-A_{12} \zeta, \\
& E_{0}=-B_{11},
\end{aligned}
$$

We shall call the tetrahedron of reference giving rise to the equation (2) the canonical tetrahedron. Every coefficient appearing in (2) is an absolute invariant of $S$ at $O$.

We find it convenient to introduce homogeneous projective coordinates by the formulas

$$
\xi=\frac{x_{1}}{x_{4}}, \quad \eta=\frac{x_{2}}{x_{4}}, \quad \zeta=\frac{x_{3}}{x_{4}} .
$$

3. Canonical form of the equation of a curve with a cusp. In this section we shall derive a canonical form of the equation of a curve with a cusp, and 
define certain osculating curves of the curve. We find these osculants useful in discussing a surface in the neighborhood of a spine.

Let $(\xi, \eta)$ be the nonhomogeneous projective coordinates of a point $P$ in a plane. Let the point $(0,0)$ be chosen at the cusp $O$ of the curve $C$, and let the side $\eta=0$ of the triangle of reference be the cuspidal tangent of $C$ at $O$. By proper choice of the side $\xi=0$, the equation of the curve $C$ may be written in the form

$$
\begin{aligned}
\eta^{2}= & \alpha_{30} \xi^{3}+\alpha_{12} \xi \eta^{2}+\alpha_{03} \eta^{3}+\alpha_{40} \xi^{4}+\alpha_{31} \xi^{3} \eta+\alpha_{22} \xi^{2} \eta^{2}+\alpha_{13} \xi \eta^{3}+\alpha_{04} \eta^{4} \\
& +\alpha_{50} \xi^{5}+\alpha_{41} \xi^{4} \eta+\alpha_{32} \xi^{3} \eta^{2}+\alpha_{23} \xi^{2} \eta^{3}+\alpha_{14} \xi \eta^{4}+\alpha_{05} \eta^{5}+\cdots, \alpha_{30} \neq 0 .
\end{aligned}
$$

If on the equation (4) of $C$ we make, in order, the transformations

$$
\begin{array}{lll}
\xi=\frac{\bar{x}}{1+p \bar{x}+q \bar{y}}, & \eta=\frac{\bar{y}}{1+p \bar{x}+q \bar{y}}, \\
\bar{x}=r x, & \bar{y}=s y,
\end{array}
$$

wherein $p, q, r, s$ are defined by the expressions

$$
\begin{aligned}
p \alpha_{30} & =\alpha_{40}+\alpha_{30} \alpha_{12}, \quad q \alpha_{30}=\alpha_{31}+\alpha_{30} \alpha_{03}, \\
\alpha_{30} r^{3} & =s^{2}, \quad r\left(\alpha_{22} \alpha_{30}+\alpha_{50}\right)=s\left(\alpha_{13} \alpha_{30}+\alpha_{41}\right),
\end{aligned}
$$

we reduce (4) to the following canonical form

$$
\begin{aligned}
y^{2}= & x^{3}+a_{12} x y^{2}+a_{03} y^{3}-a_{12} x^{4}-a_{03} x^{3} y+a_{22} x^{2} y^{2}+a_{13} x y^{3}+a_{04} y^{4} \\
& +a_{50} x^{5}+a_{41} x^{4} y+a_{32} x^{3} y^{2}+a_{23} x^{2} y^{3}+a_{14} x y^{4}+a_{05} y^{5}+\cdots
\end{aligned}
$$

wherein $a_{22}+a_{50}=a_{13}+a_{41} \neq 0$. We shall speak of the triangle giving rise to the canonical form (7) as the canonical triangle.

From the canonical form (7) we derive the equations of $C$ in the simple parametric form

$$
x=t^{2}, \quad y=t^{3}+\alpha_{7} t^{7}+\alpha_{8} t^{8}+\alpha_{9} t^{9}+\alpha_{10} t^{10}+\cdots,
$$

wherein

$$
\alpha_{7}=\alpha_{8}=\frac{1}{2}\left(a_{22}+a_{50}\right), \quad \alpha_{9}=\frac{1}{2}\left[a_{12}\left(a_{22}+a_{50}\right)+a_{04}+a_{32}+a_{60}\right], \cdots .
$$

The transformation of coordinates between the triangle of reference for the equation of the curve $C$ in the form (4) to the canonical triangle may be written in the form

$$
\xi=\frac{r x}{1+p r x+q s y}, \quad \eta=\frac{s y}{1+p r x+q s y},
$$

wherein $p, q, r, s$ are defined by the formulas (6). The inverse of transformation (9) is 


$$
x=\frac{\xi}{r(1-p \xi-q \eta)}, \quad y=\frac{\eta}{s(1-p \xi-q \eta)} .
$$

We shall find it convenient to introduce homogeneous projective coordinates by the formulas

$$
x=\frac{x_{1}}{x_{3}}, \quad y=\frac{x_{2}}{x_{3}} .
$$

4. Osculating curves of a curve at a cusp. Let $A_{p, q}^{n}$ be an algebraic curve of order $n$, with a multiple point of order $p+2$ at the cusp $O$ of $C$, two of whose multiple point tangents coincide with the cuspidal tangent $y=0$ of $C$ at $O$, and the remaining $p$ of whose tangents do not so coincide with $y=0$. Let the number of points of intersection of $A_{p, q}^{n}$ with $C$ coinciding at $O$ be denoted by $N$. We shall say that $A_{p, q}^{n}$ has $q$-point contact or $(q-1)$-order contact with $C$ at $O$ if

$$
q=N-2 p-4
$$

In the language of Bompiani we shall say that the curve $A_{p, q}^{n}$ represents the neighborhood of the $(q-1)$ st order of $C$ at $O$, and shall call $A_{p, q}^{n}$ an osculating curve of $C$ at $O$.

Writing the most general equation of a cubic with a cusp at $O$, and demanding that the power series resulting by the substitution of (8) into that equation shall lack terms in $t^{k},(k=0,1,2,3,4,5)$, we find the equation of the osculating cubic $A_{0,6}^{3}$ to be

$$
y^{2}=x^{3} .
$$

The equation of the osculating cubic gives a simple geometrical characterization of the canonical triangle. The vertex $\mathrm{O}_{2}(0,1,0)$ is the inflexion point of the cubic; the side $x_{3}=0$ is the inflexion tangent.

The neighborhoods of $C$ at $O$ of the sixth and seventh orders may be represented by the respective curves

$$
\begin{array}{ll}
A_{0,7}^{4}: & y^{2}=x^{3}+\left(a_{22}+a_{50}\right) x^{2} y^{2} ; \\
A_{1,8}^{4}: & y^{2}(x-y)=x^{4}-x^{3} y+\left(a_{22}+a_{50}\right) y^{4} .
\end{array}
$$

The unit point of the canonical triangle of reference is the intersection of the triple point tangent $y=x$ of $A_{1,8}^{4}$ with the osculating cubic $A_{0,6}^{3}$.

5. Sections of the surface $S$ through the spinal tangent. We now shall use the results of the previous sections in a discussion of a surface in the neighborhood of a spine of the nonparabolic type.

The plane which is given by 


$$
\zeta=m \eta,
$$

$m \neq 0$,

intersects the surface $S$ whose equation is (2) in a curve with a cusp at $O$ $(0,0,0,1)$. The line $\eta=\zeta=0$ is the cuspidal tangent for all of these sections. The planes $\eta=0, \zeta=0$ are the only planes through the spinal tangent which intersect the surface in curves with a triple point at $O$.

The equations of the curve of intersection of the plane (12) with the surface $S$ are

$$
\begin{aligned}
\zeta= & m \eta \\
\eta^{2}= & \alpha_{30} \xi^{3}+\alpha_{12} \xi \eta^{2}+\alpha_{03} \eta^{3}+\alpha_{40} \xi^{4}+\alpha_{31} \xi^{3} \eta+\alpha_{22} \xi^{2} \eta^{2}+\alpha_{13} \xi \eta^{3}+\alpha_{04} \eta^{4} \\
& +\alpha_{50} \xi^{5}+\alpha_{41} \xi^{4} \eta+\cdots
\end{aligned}
$$

wherein $\alpha_{30}, \alpha_{12}, \alpha_{03}$ are defined by the formulas

$$
\begin{aligned}
& m \alpha_{30}=1, \quad m \alpha_{12}=-A_{30}+B_{11} m-A_{03} m^{2}, \\
& m \alpha_{03}=A_{30}+A_{21} m+A_{12} m^{2}+A_{03} m^{3}, \quad m \alpha_{40}=-B_{11}, \\
& m \alpha_{31}=-\left(A_{21}+A_{12} m\right), \quad m \alpha_{22}=C_{20}+C_{11} m+C_{02} m^{2}, \\
& m \alpha_{13}=B_{30}+B_{21} m+B_{12} m^{2}+B_{03} m^{3} \\
& m \alpha_{04}=A_{40}+A_{31} m+A_{22} m^{2}+A_{13} m^{3}+A_{04} m^{4}, \\
& m \alpha_{50}=F_{00}, \quad m \alpha_{41}=E_{10}+E_{01} m, \cdots .
\end{aligned}
$$

The second of equations (13) is of the same form as (4). Hence we may derive the equations of the loci associated with the various neighborhoods of the sections of $S$ through the spinal tangent by the use of the transformations (9) and (10).

By this method we find that the equations of the osculating cubic of the section $C$ by the plane (12) are

$$
\zeta=m \eta, \quad m \eta^{2}=\xi^{3}-\left(A_{30}+A_{03} m^{2}\right) \xi \eta^{2}+\left(A_{30}+A_{03} m^{3}\right) \eta^{3} .
$$

The locus of the osculating cubic (15) is a cubic surface whose equation is

$$
\eta \zeta=\xi^{3}-\left(A_{30} \eta^{2}+A_{03} \zeta^{2}\right) \xi+\left(A_{30} \eta^{3}+A_{03} \zeta^{3}\right) .
$$

We shall call this cubic surface the osculating cubic surface of $S$ at $O$. The osculating cubic surface of course has a spine at $O$.

We note from the form of equations (15) that the points of inflexion of all of the osculating cubics of the sections of $S$ through the spinal tangent lie in a plane. This plane is the face $\xi=0$ of the canonical tetrahedron. The locus of the inflexion points of the osculating cubics is therefore a plane cubic whose equations are

$$
\xi=0, \quad \eta \zeta=A_{30} \eta^{3}+A_{03} \zeta^{3} .
$$


Equations (17) furnish another interpretation of the edges $\xi=\eta=0, \xi=\zeta=0$ of the canonical tetrahedron. The inflexion points of the cubic (17) lie on the edge $x_{1}=x_{4}=0$ of the canonical tetrahedron.

The inflexion tangent of the osculating cubic (15) intersects the spinal tangent in the point

$$
\left(m, 0,0,-A_{30}-A_{03} m^{2}\right) .
$$

There is established therefore a $(1,2)$ correspondence between the points on the spinal tangent and the planes through the spinal tangent. The vertex $O_{1}$ $(1,0,0,0)$ of the canonical tetrahedron is the only point to which correspond planes separating the planes $\eta=0, \zeta=0$ harmonically. There exist on the spinal tangent two points $D, D_{1}$ to each of which correspond identical planes. The vertices $O$ and $O_{1}$ separate $D, D_{1}$ harmonically.

The equations of the inflexional tangent of the osculating cubic (15) are

$$
\begin{gathered}
\zeta=m \eta, \\
\left(A_{30}+A_{03} m^{2}\right) \xi+\left(A_{30}+A_{03} m^{3}\right) \eta+m=0 .
\end{gathered}
$$

The locus of this inflexional tangent is the cubic ruled surface

$$
x_{2} x_{3} x_{4}=-\left(A_{30} x_{2}{ }^{2}+A_{03} x_{3}{ }^{2}\right) x_{1}+A_{30} x_{2}{ }^{3}+A_{03} x_{3}{ }^{3} .
$$

This ruled surface intersects the edge $x_{3}=x_{4}=0$ of the canonical tetrahedron in the point $O_{1}$ and in the point $(1,1,0,0)$. It intersects the edge $x_{2}=x_{4}=0$ in the point $O_{1}$ and in the point $(1,0,1,0)$. The point $(1,1,1,0)$ is therefore characterized. The line joining the spine $O$ to $(1,1,1,0)$ intersects the osculating cubic surface in the unit point of the canonical tetrahedron.

We may readily verify that the planes $\zeta=m \eta$ wherein $A_{30}-A_{03} m^{2}=0$ intersect the cubic surface (20) in a pair of torsal generators of that surface. The torsal generators intersect the spinal tangent in the two points $D$ and $D_{1}$ previously described.

The line $x_{2}=x_{3}=0$ evidently lies on the surface (20). The line whose parametric equations are

$$
x_{1}=1+m, \quad x_{2}=1, \quad x_{3}=m, \quad x_{4}=-\left(A_{30}+A_{03} m\right),
$$

also lies on the surface. Hence the surface (20) possesses two straight line directrices (flecnode curves).

The locus of the triple point tangent of the curve $A_{1,8}^{4}$ having eight-point contact with the section of $S$ at $O$ is a cubic cone whose equation is

$$
\begin{aligned}
B_{30} \eta^{3}+\left(B_{21}+E_{10}\right) \eta^{2} \zeta+ & \left(B_{12}+E_{01}\right) \eta \zeta^{3}+B_{03} \zeta^{3} \\
& =\left[C_{20} \eta^{2}+\left(C_{11}+F_{00}\right) \eta \zeta+C_{02} \zeta^{2}\right] \xi .
\end{aligned}
$$


The vertex of this cone is of course the spine $O$. The spinal tangent is a singular line; the planes

$$
C_{20} \eta^{2}+\left(C_{11}+F_{00}\right) \eta \zeta+C_{02} \zeta^{2}=0
$$

are the singular tangent planes along the line. The surface (21) intersects the face $x_{4}=0$ of the canonical tetrahedron in a cubic curve which has a double point at $O_{1}$, and whose inflexions lie on the line $x_{1}=x_{4}=0$.

Loci associated with other algebraic curves representing neighborhoods of higher order could be considered, but we shall carry this discussion no further.

6. The parabolic case. In this section we discuss briefly the geometry of a surface in the neighborhood of a spine of the parabolic type.

By proper choice of the tetrahedron of reference the equation of a surface possessing such a spine may be written in the form

(22) $\eta^{2}=A_{3}+B_{2} \xi+C_{1} \xi^{2}+D_{0} \xi^{3}+A_{4}+B_{3} \xi+C_{2} \xi^{2}+D_{1} \xi^{3}+E_{0} \xi^{4}+\cdots$, wherein

$$
\begin{aligned}
& A_{3}=A_{30} \eta^{3}+A_{21} \eta^{2} \zeta-A_{03} \eta \zeta^{2}+A_{03} \zeta^{3}, \quad A_{03} \neq 0, \\
& B_{2}=B_{20} \eta^{2}-A_{03} \zeta^{2}, \\
& C_{1}=0 \\
& D_{0}=1 \\
& A_{4}=A_{40} \eta^{4}+A_{31} \eta^{3} \zeta+A_{22} \eta^{2} \zeta^{2}+A_{13} \eta \zeta^{3}+A_{04} \zeta^{4}, \\
& B_{3}=B_{30} \eta^{3}+B_{21} \eta^{2} \zeta+B_{12} \eta \zeta^{2}+B_{03} \zeta^{3}, \\
& C_{2}=C_{20} \eta^{2}+C_{11} \eta+C_{02} \zeta^{2}, \\
& D_{1}=-A_{30} \eta-A_{21} \zeta \\
& E_{0}=-B_{2 c}
\end{aligned}
$$

The plane $\eta=0$ intersects the surface $S$ in a curve with a triple point at $O$. The triple point tangents are determined by the cubic form

$$
\xi^{3}-A_{03} \zeta^{2}(\xi-\zeta)=0 .
$$

The interpretation of the condition $A_{03} \neq 0$ is evident. The line $\eta=\zeta=0$ is an arbitrary line in the plane $\eta=0$.

The section of the surface $S$ through $O(0,0,0)$ by the plane $\zeta=m \eta$ has the equations

$$
\begin{aligned}
\zeta= & m \eta \\
\eta^{2}= & \xi^{3}+\alpha_{12} \xi \eta^{2}+\alpha_{03} \eta^{3}+\alpha_{40} \xi^{4}+\alpha_{31} \xi^{3} \eta+\alpha_{22} \xi^{2} \eta^{2}+\alpha_{13} \xi \eta^{3} \\
& +\alpha_{04} \eta^{4}+\cdots,
\end{aligned}
$$


wherein

$$
\begin{aligned}
& \alpha_{12}=B_{20}-A_{03} m^{2}, \\
& \alpha_{03}=A_{30}+A_{21} m-A_{03} m^{2}+A_{03} m^{3}, \quad \alpha_{40}=-B_{20}, \\
& \alpha_{31}=-A_{30}-A_{21} m, \quad \alpha_{22}=C_{20}+C_{11} m+C_{02} m^{2}, \\
& \alpha_{13}=B_{30}+B_{21} m+B_{12} m^{2}+B_{03} m^{3} \\
& \alpha_{04}=B_{40}+B_{31} m+B_{22} m^{2}+B_{13} m^{3}+B_{04} m^{4}
\end{aligned}
$$

If use be made of formula (10) and the definitions of $p, q, r, s$ occurring therein, we may show that the equations of the osculating cubic of the section (24) of $S$ are

$$
\begin{aligned}
\zeta & =m \eta, \\
\eta^{2} & =\xi^{3}-A_{03} m^{2} \eta^{2}[\xi-(1+m) \eta] .
\end{aligned}
$$

Equations (25) show that the inflexion points of the osculating cubics of all sections through a given arbitrary spinal tangent $\eta=\zeta=0$ lie on the fixed plane $\xi=0$.

The locus of the osculating cubic is the cubic surface with the equation

$$
\eta^{2}=\xi^{3}-A_{03} \zeta^{2}(\xi-\eta-\zeta) .
$$

The inflexional tangent of the curve (25) intersects the line $\eta=\zeta=0$ in a point whose homogeneous coordinates are $\left(1,0,0,-A_{03} m^{2}\right)$. As in the nonparabolic case, there is thus established a $(1,2)$ correspondence between the points on $\eta=\zeta=0$ and the planes through that spinal tangent. The point $O_{1}$ $(1,0,0,0)$ is the only point on the line to which correspond identical planes. Moreover the planes corresponding to the points on $\eta=\zeta=0$ are paired in involution; the double planes of the involution are the planes $\eta=0, \zeta=0$.

The line $\xi=\zeta=0$ intersects the surface (26) in $O$ and in the vertex $O_{2}$ $(0,1,0,0)$ of the tetrahedron of reference.

The inflexion point of the cubic (25) has the homogeneous coordinates

$$
x_{1}=0, \quad x_{2}=1, \quad x_{3}=m, \quad x_{4}=A_{03} m^{2}(1+m) .
$$

The locus of this point passes through the vertex $(0,1,0,0)$, and the tangent to the locus at this vertex is the line $\xi=\zeta=0$.

The inflexional tangent of the osculating cubic (25) has the equations

$$
\begin{gathered}
\zeta=m \eta, \\
A_{03} m^{2}[\xi-(1+m) \eta]+1=0 .
\end{gathered}
$$

The locus of this inflexional tangent is the cubic ruled surface

$$
x_{2}^{2} x_{4}=-A_{03}\left[x_{1}+x_{2}-x_{3}\right] x_{3}^{2} .
$$


The edge $x_{3}=x_{4}=0$ lies on this cubic; the edge $x_{2}=x_{4}=0$ cuts the cubic in the points $O_{1}$ and $(1,0,1,0)$. The edge $x_{1}=x_{4}=0$ cuts the cubic in the point $O_{2}$ and $(0,1,1,0)$. The point $(1,1,1,0)$ is therefore characterized.

The osculating cubic of the plane section of $S$ by the plane $\zeta=0$ has the equation

$$
\zeta=0, \quad \eta^{2}=\xi^{3} .
$$

The projection of this cubic from the vertex $(0,0,1,0)$ is the cubic cone

$$
x_{2}^{2} x_{4}=x_{1}^{3} \text {. }
$$

The line joining $O$ to $(1,1,1,0)$ intersects this cubic cone in the unit point of the tetrahedron of reference.

Hence the tetrahedron of reference giving rise to the expansion (22) and the unit point of the system have been geometrically characterized. Other properties of the surface could be obtained by considering the loci or envelopes of the various curves and surfaces associated with the sections of $S$ through the arbitrary spinal tangent $t$ in the plane $\eta=0$ by allowing $t$ to vary in that plane. We shall refrain from discussing these details in this paper.

Michigan State College,

East Lansing, Mich. 